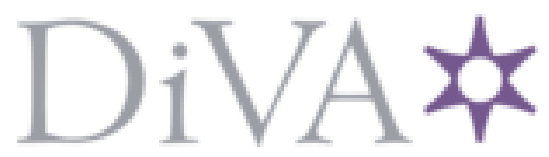

http://www.diva-portal.org

This is the published version of a paper published in Physics of Plasmas.

Citation for the original published paper (version of record):

Karimov, A., Yu, M., Stenflo, L. (2014)

Expansion of a cold non-neutral plasma slab.

Physics of Plasmas, 21(12)

http://dx.doi.org/10.1063/1.4903879

Access to the published version may require subscription.

N.B. When citing this work, cite the original published paper.

Permanent link to this version:

http://urn.kb.se/resolve?urn=urn:nbn:se:umu:diva-104153 


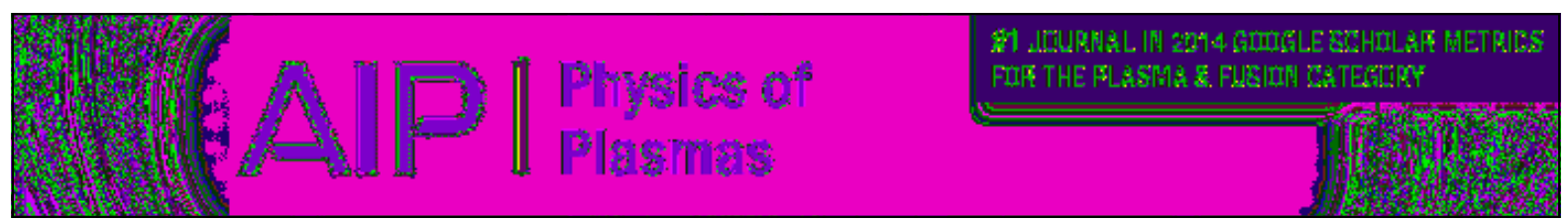

\section{Expansion of a cold non-neutral plasma slab}

A. R. Karimov, M. Y. Yu, and L. Stenflo

Citation: Physics of Plasmas 21, 122304 (2014); doi: 10.1063/1.4903879

View online: http://dx.doi.org/10.1063/1.4903879

View Table of Contents: http://scitation.aip.org/content/aip/journal/pop/21/12?ver=pdfcov

Published by the AIP Publishing

\section{Articles you may be interested in}

A water bag model of driven phase space holes in non-neutral plasmas

Phys. Plasmas 15, 082110 (2008); 10.1063/1.2969738

Drift resonance in high density non-neutral plasmas

Phys. Plasmas 13, 053113 (2006); 10.1063/1.2199227

Simple modes of thin oblate non-neutral plasmas

Phys. Plasmas 10, 1239 (2003); 10.1063/1.1569488

Continuous spectrum of a non-neutral plasma column

Phys. Plasmas 4, 895 (1997); 10.1063/1.872184

Damped diocotron quasi-modes of non-neutral plasmas and inviscid fluids

Phys. Plasmas 4, 53 (1997); 10.1063/1.872497

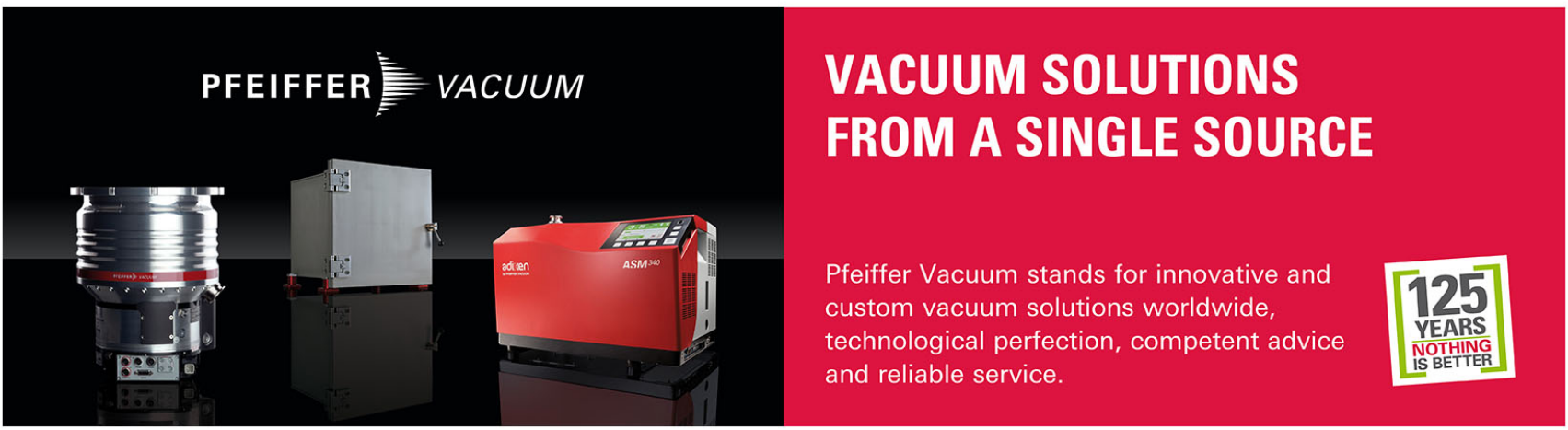




\title{
Expansion of a cold non-neutral plasma slab
}

\author{
A. R. Karimov, ${ }^{1,2}$ M. Y. Yu, ${ }^{3,4, a)}$ and L. Stenflo ${ }^{5,6}$ \\ ${ }^{1}$ Institute for High Temperatures, Russian Academy of Sciences, Izhorskaya 13/19, Moscow 127412, \\ Russia \\ ${ }^{2}$ Department of Electrophysical Facilities, National Research Nuclear University MEPhI, \\ Kashirskoye shosse 31, Moscow 115409, Russia \\ ${ }^{3}$ Institute for Fusion Theory and Simulation, Department of Physics, Zhejiang University, \\ 310027 Hangzhou, China \\ ${ }^{4}$ Institut für Theoretische Physik I, Ruhr-Universität Bochum, D-44780 Bochum, Germany \\ ${ }^{5}$ Department of Physics, Linköping University, SE-58183 Linköping, Sweden \\ ${ }^{6}$ Department of Plasma Physics, Umeå University, SE-90187 Umea, Sweden
}

(Received 13 October 2014; accepted 21 November 2014; published online 12 December 2014)

Expansion of the ion and electron fronts of a cold non-neutral plasma slab with a quasi-neutral core bounded by layers containing only ions is investigated analytically and exact solutions are obtained. It is found that on average, the plasma expansion time scales linearly with the initial inverse ion plasma frequency as well as the degree of charge imbalance, and no expansion occurs if the cold plasma slab is stationary and overall neutral. However, in both cases, there can exist prominent oscillations on the electron front. (C) 2014 AIP Publishing LLC.

[http://dx.doi.org/10.1063/1.4903879]

\section{INTRODUCTION}

A plasma is non-neutral if its total charge is sufficiently different from null, such that the resulting space charge electrostatic field plays crucial roles in the dynamics of the system. ${ }^{1}$ Non-neutral plasmas can occur in natural and laboratory environments. ${ }^{1,2}$ Aside from single-species plasmas, ${ }^{1-5}$ they can be at the edge, or boundary, regions of neutral plasmas in the presence of walls, inertial or external forces, etc. ${ }^{1,6-9}$ They can also be formed in evolving systems, such as in the expansion of neutral plasmas, ${ }^{10-14}$ around intense charged-particle beams or laser pulses propagating in gases or plasmas, ${ }^{15,16}$ on the interfaces of materials, ${ }^{17,18}$ in plasmas containing highly charged dust grains, ${ }^{11,19,20}$ etc.

Although a plasma tends to remain charge neutral, ${ }^{6}$ nonneutral edge regions are often formed because of the large difference in the electron and ion masses. ${ }^{1,6}$ Also for the same reason, in warm or hot plasmas, the time and space scales of the non-neutral regions are usually small, of the order of the inverse electron plasma frequency and Debye length, respectively. As a result, such edge regions usually do not affect bulk-plasma phenomena unless they are unstable or cause fast particle or energy loss. However, in cold plasmas, the effect of the non-neutral region can often be important or even dominant.

In this paper, we consider the hydrodynamic expansion of the electron and ion fronts of a cold plasma slab containing a quasineutral core bounded on both sides by a layer with only ions. As with most expansions into vacuum, the problem is necessarily fully nonlinear, ${ }^{21}$ so that no perturbation analysis can be used.

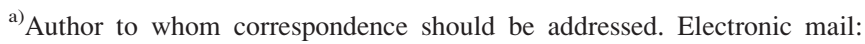
myyu@zju.edu.cn
}

\section{FORMULATION}

In view of the symmetry of the slab, we shall assume that the plasma and vacuum are in the half space $x>0$. The cold plasma has a center region $\left(0 \leq x \leq x_{e}(0)\right)$ with equal numbers of ions and electrons, bounded by an ions-only edge layer $\left(x_{e}(0) \leq x \leq x_{i}(0)\right)$ and vacuum $\left(x>x_{i}(0)\right)$, as shown in Fig. 1. We are interested in the evolution of the fronts $x_{i}(t)$ and $x_{e}(t)$ of the cold ion and electron fluids as they expand into the vacuum.

The hydrodynamic conservation equations for the cold fluid plasma are

$$
\begin{aligned}
& \frac{\partial n_{s}}{\partial t}+\frac{\partial}{\partial x}\left(n_{s} v_{s}\right)=0, \\
& \frac{\partial v_{s}}{\partial t}+v_{s} \frac{\partial v_{s}}{\partial x}=\frac{q_{s}}{m_{s}} E, \\
& \frac{\partial E}{\partial x}=4 \pi e\left(n_{i}-n_{e}\right),
\end{aligned}
$$

where $n_{s}, v_{s}, q_{s}= \pm e$, and $m_{s}$ are the density, velocity, charge, and mass, respectively, of the particle species $s=i$, $e$, and $E$ is the electrostatic field.

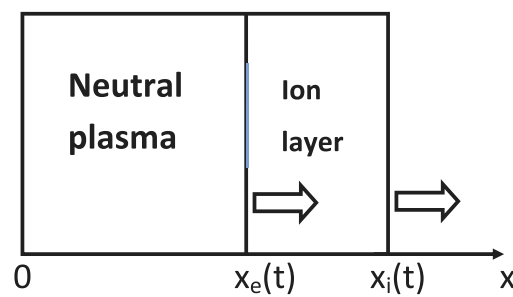

FIG. 1. Sketch of a non-neutral slab consisting of a quasineutral core plasma with equal number of ions and electrons, and an edge layer containing only ions. The electron and ion densities are thus not the same in the core region, which has a net charge separation field. The behavior of the ion front $x_{i}(t)$ and electron front $x_{e}(t)$ is investigated. 
Conservation of the total number

$$
N_{s}\left(x_{s}(t), t\right)=\int_{0}^{x_{s}(t)} n_{s}\left(t, x^{\prime}\right) d x^{\prime}
$$

of $s$ particle species in the plasma requires $d_{t} N_{s}=0$, or

$$
n_{s} \frac{\partial x_{s}}{\partial t}+\int_{0}^{x_{s}(t)} \frac{\partial n_{s}}{\partial t} d x^{\prime}=0
$$

which in view of (1) and the boundary condition $\left[n_{s} v_{s}\right]_{x=0}=0$ can be written as

$$
n_{s} \frac{\partial x_{s}}{\partial t}-\left[n_{s} v_{s}\right]_{x=x_{s}}=0 .
$$

Furthermore, we have

$$
\frac{\partial x_{s}}{\partial t}=v_{s}\left(t, x_{s}(t)\right)
$$

Similarly, the total momentum

$$
P_{s}\left(x_{s}(t), t\right)=m_{s} \int_{0}^{x_{s}(t)} n_{s}\left(t, x^{\prime}\right) v_{s}\left(t, x^{\prime}\right) d x^{\prime}
$$

of the $s$ particles in the slab satisfies

$$
\frac{\partial P_{s}}{\partial t}=m_{s} \frac{\partial x_{s}}{\partial t}\left[n_{s} v_{s}\right]_{x=x_{s}}+m_{s} \int_{0}^{x_{s}(t)} \frac{\partial}{\partial t}\left(n_{s}\left(t, x^{\prime}\right) v_{s}\left(t, x^{\prime}\right)\right) d x^{\prime} .
$$

Substitution of (2) and (7) into (9) leads to

$$
\frac{\partial P_{s}}{\partial t}=q_{s} n_{s} \int_{0}^{x_{s}(t)} E\left(x^{\prime}\right) d x^{\prime} .
$$

\section{EQUATIONS FOR THE ELECTRON AND ION FRONTS}

We look for solutions of (1)-(3) in the form

$$
n_{s}=n_{s}(t) \quad \text { and } \quad v_{s}=\frac{\dot{x}_{s}(t)}{x_{s}(t)} x
$$

where the overhead dot denotes derivative with respect to $t$. The Ansatz (11) is as expected not unique and its origin is discussed in more detail in Appendix A. Substituting (11) into (4), we get for the densities

$$
n_{s}=N_{s 0} / x_{s},
$$

where the total number $N_{s 0}$ of $s$ particles is given by

$$
N_{s 0}=\int_{0}^{x_{s 0}} n_{s}\left(t=0, x^{\prime}\right) d x^{\prime}=n_{s 0} x_{s 0},
$$

and $x_{s 0}=x_{s}(0)$. One can thus verify that (1) is satisfied.

Inserting (12) into (3), we obtain

$$
E=4 \pi e\left\{\begin{array}{lll}
N_{i 0} \frac{x}{x_{i}}-N_{e 0} \frac{x}{x_{e}} & \text { for } & 0 \leq x \leq x_{e}, \\
N_{i 0} \frac{x}{x_{i}}-N_{e 0} & \text { for } & x_{e} \leq x \leq x_{i},
\end{array}\right.
$$

which we see is continuous at $x=x_{e}(t)$.

Combining (14), (11), and (3), one obtains the governing equations for $x_{e}(t)$ and $x_{i}(t)$

$$
\begin{gathered}
\frac{d^{2} x_{e}}{d t^{2}}=\frac{4 \pi e^{2}}{m_{e}}\left(\frac{N_{e 0}}{x_{e}}-\frac{N_{i 0}}{x_{i}}\right) x_{e}, \\
\frac{d^{2} x_{i}}{d t^{2}}=\frac{4 \pi e^{2}}{m_{i}}\left(N_{e 0} \frac{x_{e}}{x_{i}}+N_{i 0}-2 N_{e 0}\right) .
\end{gathered}
$$

Using (13) and the normalization $\tau=t \omega_{p i}, \alpha_{e}=x_{e} / x_{e 0}$, and $\alpha_{i}=x_{i} / x_{i 0}$, where $\omega_{p i}=\left(4 \pi e^{2} n_{i 0} / m_{i}\right)^{1 / 2}$ is the ion plasma frequency, we can rewrite (15) and (16) as

$$
\delta \frac{d^{2} \alpha_{e}}{d \tau^{2}}=\nu-\frac{\alpha_{e}}{\alpha_{i}},
$$

$$
\frac{d^{2} \alpha_{i}}{d \tau^{2}}=\nu \lambda^{2} \frac{\alpha_{e}}{\alpha_{i}}+1-2 \nu \lambda,
$$

where $\delta=m_{e} / m_{i}, \nu=n_{e 0} / n_{i 0}$, and $\lambda=x_{e 0} / x_{i 0}$.

Equations (17) and (18) are valid for the electron and ion fronts of neutral as well as non-neutral cold plasma slabs. Together with the initial conditions $\alpha_{e}(0)=\alpha_{i}(0)=1$ and $\dot{\alpha}_{e}(0)=\dot{\alpha}_{i}(0)=0$, where the overhead dot now denotes derivative with respect to $\tau$, they govern the evolution of the ion and electron fronts of the cold plasma slab in terms of the parameters $\nu$ and $\lambda$. In general, these equations cannot be solved analytically. Since they have no fixed point for any $\nu$ and $\lambda$, there is also no stationary solution.

\section{MOTION OF AN AVERAGED PLASMA FRONT}

From (17) and (18), one can obtain the evolution equation for the weighted-average front $\alpha_{\mathrm{avg}}=\delta \nu \lambda^{2} \alpha_{e}+\alpha_{i}$ of the plasma slab

$$
\frac{d^{2} \alpha_{\mathrm{avg}}}{d \tau^{2}}=(\xi-1)^{2}
$$

where $\xi=\nu \lambda=N_{e 0} / N_{i 0}$, so that $\xi-1$ is a measure of the overall charge imbalance of the plasma slab. We see that the weighted-average front is driven by a constant force proportional to the square of the charge imbalance and $\mid N_{e 0} / N_{i 0}$ $-1 \mid / \omega_{p i}$ is the time scale of the expansion. The straightforward solution

$$
\alpha_{\mathrm{avg}}=(1 / 2)(\xi-1)^{2} \tau^{2}+\delta \nu \lambda^{2}+1
$$

is analogous to the parabolic time dependence of an initially stationary object rising under negative gravity. Although the rate of expansion increases indefinitely with $\tau$, the particle densities become rapidly very small because of total number conservation.

If we assume initial charge neutrality $(\xi=1)$, the averaged front of the plasma slab would remain stationary (recall 
that we have assumed zero initial velocities for the ion and electron, and therefore the averaged, fronts). However, as we shall see below, oscillations in the fronts can still take place. In contrast, for a one-component plasma, say, of only ions, Eq. (18) reduces to $\ddot{\alpha}_{i}=1$. Accordingly, a stationary cold ion slab expands linearly in time at the scale of the inverse plasma frequency.

The effect of finite electron-to-ion mass ratio $\delta$, a very small quantity, is not obvious in the evolution of the weighted-average plasma front. If we set $\delta=0$ in (20), we obtain the trajectory of the ion front of a plasma slab, with the massless electron fluid frozen to it. This scenario is quite different from that of a hot plasma, where the electrons respond adiabatically to the space charge field and expansion takes place even if the initial plasma slab is neutral and stationary. ${ }^{10-12}$ However, as we shall see, the effect of the small $\delta$ can still be important, especially at the front of the expanding cold plasma.

\section{MOTION OF THE ELECTRON AND ION FRONTS}

To see the evolution of the fronts of the ion and electron fluids, we solve the coupled equations (17) and (18) numerically for $\delta=1 / 1837$. Typical results for different values of the parameters $\nu$ and $\lambda$ are shown in Fig. 2. One can see that in general, the electron front tends to oscillate prominently. Such surface oscillations, or surface plasma waves, are ubiquitous at the boundaries of plasmas, but they are evanescent in the surface-normal directions and usually weak. ${ }^{22-24}$ However, Fig. 2 shows that in the expansion of a cold plasma slab, the oscillations of the normalized electron front can be of large amplitude and are stably modulated, and, depending of the initial ion and electron densities and the size of the edge ion layer, can be either in front of or behind the ion front (which in the present non-perturbative analysis actually also oscillates, but at very small amplitude and long wave length because of the large ion mass). This is to be expected since the charge separation field at the plasma edge can be large in the edge ion layer, or the initial charge nonneutrality, is large.

The last row in Fig. 2 shows the evolution of neutral $(\xi=1)$ plasma slabs. We see that even if the initial electron density is high, but if the overall plasma slab is neutral, both the electron and ion fronts do not expand. However, stable large-amplitude oscillations of the electron front (as mentioned, also the ion front, but extremely weak) can exist. These oscillations can be identified as nonlinear symmetric standing surface waves of the plasma slab. ${ }^{23}$

The limit $\xi=0$ corresponds to an extremely thin $(\lambda \rightarrow 0)$ central plasma layer, or surface charges. As a result we have $\alpha_{i}(\tau)=1+\tau^{2} / 2$, and $\alpha_{e}(\tau)$ can be expressed in terms of a hypergeometric function. Numerical results from solving (17) and (18) for this case are shown in Fig. 3. We see that both fronts still expand like in the cases shown in Fig. 2. However, the oscillations in $\alpha_{e}$ are of much longer periods and smaller amplitudes.

\section{DISCUSSIONS}

Even though the governing equations (17) and (18) cannot be solved analytically, one can nevertheless obtain a few basic relations that should be helpful for understanding the behavior, and thus the physics, of the numerical results on the slab expansion. From (17), we have $\alpha_{e}=\alpha_{i}\left(\nu-\delta \ddot{\alpha}_{e}\right)$, which together with (18) yields
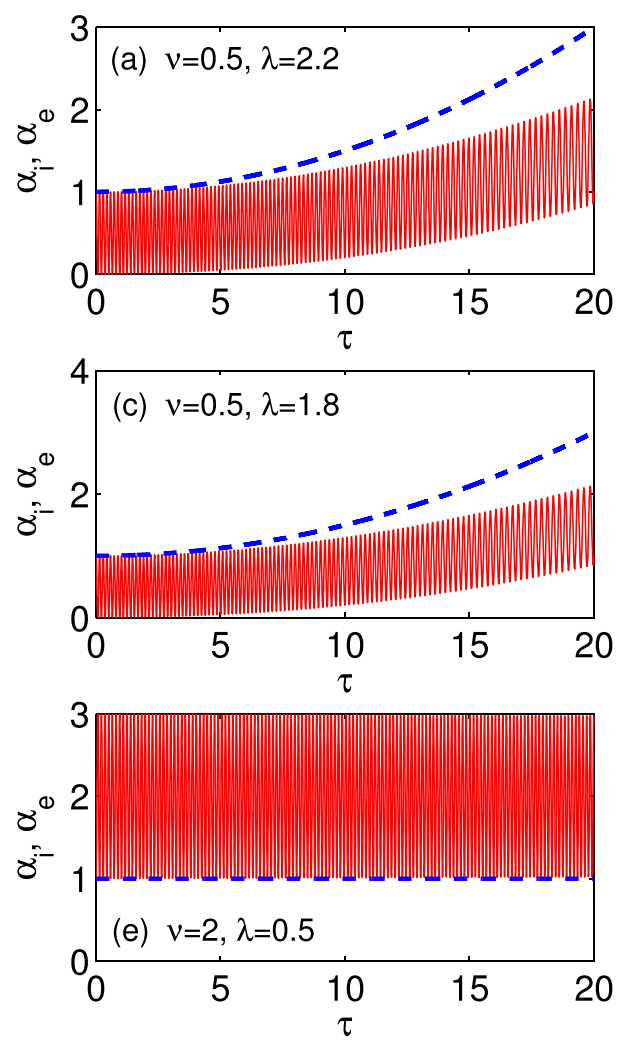
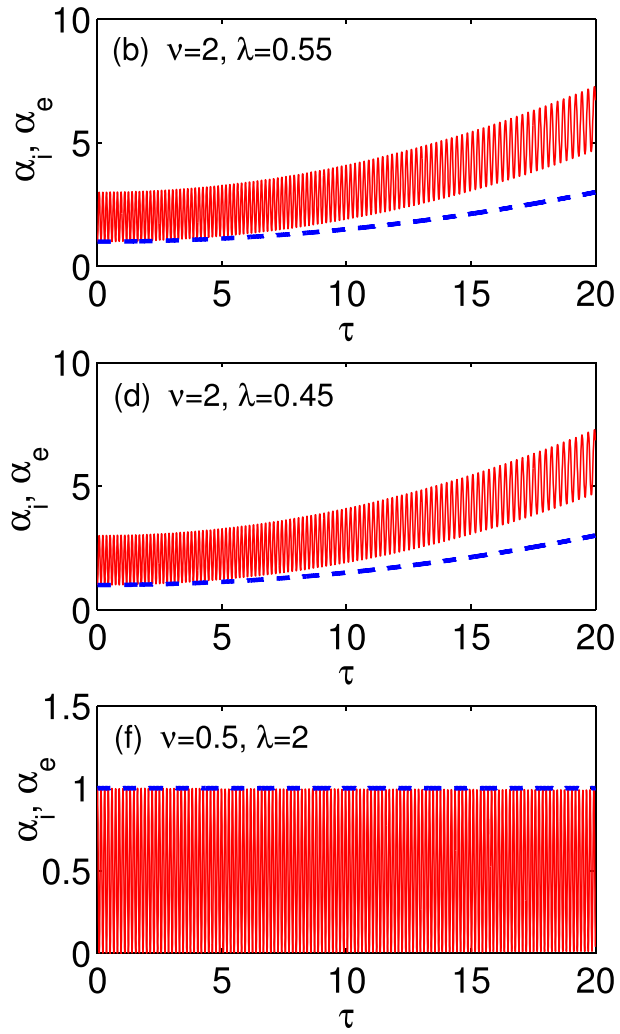

FIG. 2. Evolution of $\alpha_{i}$ (blue dashed curves) and $\alpha_{e}$ (red solid curves) for different values of $\nu$ and $\lambda$. Note that in (e) and (f), the cold plasma layer is neutral $(\xi=\nu \lambda=1)$ and there is no expansion. 


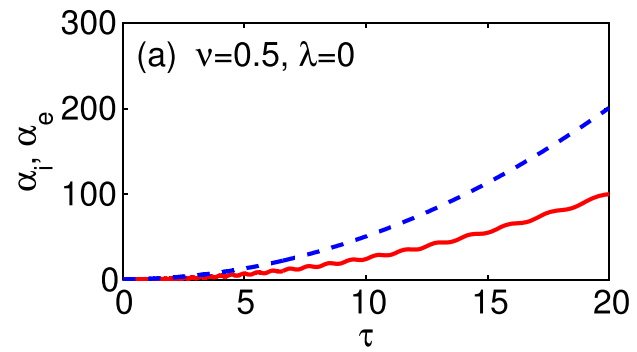

$$
\frac{\partial^{2} \alpha_{i}}{\partial \tau^{2}}=(1-\nu \lambda)^{2}-\delta \nu \lambda^{2} \frac{\partial^{2} \alpha_{e}}{\partial \tau^{2}} .
$$

Integrating (21) once, we obtain

$$
\frac{\partial \alpha_{i}}{\partial \tau}=(1-\nu \lambda)^{2} \tau-\delta \nu \lambda^{2} \frac{\partial \alpha_{e}}{\partial \tau}
$$

where the initial condition $\dot{\alpha}_{s}(0)=0$ has been applied.

We see that for $\xi \neq 1$, the fronts $\alpha_{e}$ and $\alpha_{i}$ cannot simultaneously reach extremum during the expansion: they always have different critical points. In fact, integration of (22) and using the initial condition $\dot{\alpha}_{e, i}(0)=1$ gives

$$
\alpha_{i}(\tau)=1+(1-\nu \lambda)^{2} \tau^{2} / 2-\delta \nu \lambda^{2}\left(\alpha_{e}(\tau)-1\right),
$$

which relates $\alpha_{e}(\tau)$ and $\alpha_{i}(\tau)$. Even though the former can be larger than the latter, its behavior can only weakly affect the latter since $\delta \ll 1$. Moreover, as already mentioned, the ion front expands mainly parabolically in time and for fully neutral slabs, it remains nearly stationary. These results are consistent with that for the weighted-average front $\alpha_{\mathrm{avg}}(\tau)$ of the expansion considered in Sec. IV.

One can obtain analytical expressions for the slowly varying envelopes of $\alpha_{s}(t)$ by taking the limit $\delta \ddot{\alpha}_{e} \rightarrow 0$. Equation (17) then becomes $\alpha_{e}(t)=\nu \alpha_{i}(t)$. Substituting the latter into (23), one can obtain the parabolic envelopes

$$
\bar{\alpha}_{i}(t)=\frac{1+\delta \nu \lambda^{2}}{1+\delta \nu^{2} \lambda^{2}}+\frac{(1-\nu \lambda)^{2}}{1+\delta \nu^{2} \lambda^{2}} \frac{t^{2}}{2} \quad \text { and } \quad \bar{\alpha}_{e}(t)=\nu \bar{\alpha}_{i}(t)
$$

where the overhead bar denotes the slowly varying envelope of the expansion front $\alpha_{s}(t)$. Comparing (17) and (18), one can easily demonstrate that $\bar{\alpha}_{i}(t) \sim \alpha_{i}(t)$, as found in the numerical solutions.

As pointed out earlier, Fig. 2 shows that the expansion can be led by either the ion or the electron front. We can observe from the cases shown that $\alpha_{e} \geq \alpha_{i}$ for $\nu>1$, and vice versa for $\nu<1$. That is, whether the expansion is led by the electron or ion front depends on the direction of the initial electric field in the slab. Although this argument is physically reasonable, its rigorous justification turns out to be not obvious. A proof that this conclusion is indeed valid is given in Appendix B. It should also be mentioned that since we have considered only the $x \geq 0$ half space, left-right asymmetric oscillations ${ }^{22-24}$ on the expansion fronts have been precluded.

\section{CONCLUSION}

We have considered the evolution of an initially stationary one-dimensional cold plasma slab consisting of a neutral central core bounded on both sides by layers containing only ions. The dynamics of the fronts of the ion and electron fluids are followed. As expected, a cold fully neutral stationary slab does not expand. However, large amplitude surface plasma oscillations can appear on the electron front. In contrast, nonneutral slabs can expand into the vacuum around it. The expansion is initiated by the space charge field in the slab, and also accompanied by large-amplitude surface oscillations of the electron fluid. The evolution of the weighted-average front has in general a parabolic time dependence, on the time scale $\left|N_{e 0} / N_{i 0}-1\right| \omega_{p i}^{-1}$ determined by the initial charge imbalance and ion plasma frequency. However, the characteristics of the ion and electron fronts, such as their relative locations and the oscillations, depend on the charge imbalance as well as the thickness of the bounding ion layer. Moreover, although here we have considered uniform electron and ion densities, the relation (A2) in Appendix A does not preclude solutions with nonuniform density and velocity profiles similar to that found for one-component ${ }^{25}$ and force-free ${ }^{26}$ plasmas.

Our findings should be helpful in identifying or interpreting cold-plasma expansion phenomena found in space and laboratory experiments, as well as numerical simulations. ${ }^{27-34}$ Finally, it should be mentioned that the method of analysis used here is similar to the Karman momentum integral method for treating moving boundary layers. ${ }^{35}$ Moreover, higher-dimensional solutions, whose base structures have to be found by trial and error, ${ }^{37,38}$ can also exist.

\section{ACKNOWLEDGMENTS}

We would like to dedicate our work to Professor A. S. Pleshanov [28.11.1930-24.07.2006] from whom some of the ideas in the present problem originate, as well as thank Yawei Hou and Youmei Wang for useful discussions. This work was supported by the National Natural Science Foundation of China (11205194, 11247007, 11374262), ITER-CN (2013GB104004), and the Open Fund of the State Key Laboratory of High Field Laser Physics at SIOM.

\section{APPENDIX A: THE SOLUTION STRUCTURE}

In our paper, the solution structure (11) has been put forward as an Ansatz and then shown a posteriori to be valid. Here, we give a mathematical argument that it can indeed be a possible solution structure. 
In the Lagrangian frame, using the expression (4) for the total number of particles per unit length in the region $0 \leq x^{\prime} \leq x$, where $x$ is the space coordinate, Eq. (1) can be written as

$$
\frac{\partial N_{s}}{\partial t}+v \frac{\partial N_{s}}{\partial x}=0
$$

That is, $N_{s}(x, t)$ is constant in the coordinate system moving with the velocity

$$
v_{s}(x, t)=-\frac{\partial N_{s} / \partial t}{\partial N_{s} / \partial x},
$$

where instead of $N$ one can also use any function whose convective derivative vanishes (A1). ${ }^{42-44}$ In particular, solutions with nonuniform densities, and therefore velocity profiles, can be expected to exist.

Since in our problem the density $n_{s}$ is spatially uniform, we have $\partial N_{s} / \partial x=n_{s}$ and $\partial N_{s} / \partial t=x \dot{n}$. It then follows from (5) that $\dot{n}_{s}=-\dot{x}_{s} n_{s} / x_{s}$. Substituting these relations into (A2) leads directly to (11). Similar approaches have been used by Stanyukovich $^{40}$ for studying astrophysical oscillations, and Amiranashvili et l. $^{24}$ and Dubin ${ }^{41}$ for studying nonlinear oscillations in non-neutral oblate and regular spheroidal plasmas.

\section{APPENDIX B: CHAPLYGIN THEOREM}

Making use of the mathematical properties of (17) and (18) and a Chaplygin theorem ${ }^{36}$ on inequalities, here we consider the observed relation between $\alpha_{e} / \alpha_{i}$ and $\nu$.

Substituting (23) into (17), we can obtain $\varepsilon\left(\alpha_{e}\right)=0$, where the functional $\varepsilon\left(\alpha_{e}\right)$ is given by

$$
\varepsilon\left(\alpha_{e}\right)=\delta \ddot{\alpha}_{e}-\nu+\alpha_{e}\left[1+\frac{1}{2}(1-\lambda \nu)^{2} \tau^{2}-\delta \nu \lambda^{2}\left(\alpha_{e}-1\right)\right]^{-1} .
$$

Since $\alpha_{i}(\tau)>0$, it follows that $\alpha_{i}(\tau) \leq y(\tau)$, where $y(\tau)=1+\delta \nu \lambda^{2}+(1-\nu \lambda)^{2} \tau^{2} / 2$. get

Replacing $\alpha_{e}(\tau)$ by the simpler function $y(\tau)$ in (B1), we

$$
\varepsilon(y)=\delta(1-\lambda \nu)^{2}-\nu+\left(1-\delta \nu \lambda^{2}\right)^{-1} .
$$

Accordingly, if

$$
\nu \geq \delta(1-\lambda \nu)^{2}+\left(1-\delta \nu \lambda^{2}\right)^{-1},
$$

then $\varepsilon(y) \leq 0$, and from the Chaplygin theorem ${ }^{36}$ one can conclude that $y(t) \leq \alpha_{e}$, or $\alpha_{i} \leq \alpha_{e}$. Since $\delta \ll 1$, the condition (B3) is practically the same as $\nu>1$.

For the opposite case $\nu<1$, in view of $\delta \ll 1$, we have $\delta \ddot{\alpha}_{e} \ll 1$. From (18), we can then obtain

$$
\alpha_{i}=\frac{\alpha_{e}}{\nu-\delta \ddot{\alpha}_{e}} \approx \frac{\alpha_{e}}{\nu},
$$

from which it follows that $\alpha_{e}<\alpha_{i}$ for $\nu<1$. Note that in this case, the fronts $\alpha_{e}$ and $\alpha_{i}$ never cross. A similar result has been obtained for the expansion of collisionless hot Vlasov plasmas. $^{39}$

${ }^{1}$ R. C. Davidson, Physics of Non-Neutral Plasmas (Addison-Wesley, Redwood City, CA, 1990).

${ }^{2}$ J. H. Malmberg and J. S. DeGrassie, Phys. Rev. Lett. 35, 577 (1975)

${ }^{3}$ J. J. Bollinger and D. J. Wineland, Phys. Rev. Lett. 53, 348 (1984).

${ }^{4}$ R. G. Greaves, M. D. Tinkle, and C. M. Surko, Phys. Plasmas 1, 1439 (1994).

${ }^{5} \mathrm{~F}$. Anderegg, in Physics With Trapped Charged Particles: Lectures from the Les Houches Winter School (Imperial College Press, London, 2013).

${ }^{6}$ F. F. Chen, Introduction to Plasma Physics (Plenum, New York, 1974).

${ }^{7}$ D. Durkin and J. Fajans, Phys. Fluids 12, 289 (2000).

${ }^{8}$ D. H. E. Dubin and T. M. O'Neil, Rev. Mod. Phys. 71, 87 (1999).

${ }^{9}$ X.-P. Huang, F. Anderegg, E. M. Hollmann, C. F. Driscoll, and T. M. O’Neil, Phys. Rev. Lett. 78, 875 (1997).

${ }^{10} \mathrm{Ch}$. Sack and H. Schamel, Phys. Rep. 156, 311 (1987).

${ }^{11}$ H. Luo and M. Y. Yu, Phys. Fluids B 4, 1122 (1992).

${ }^{12}$ P. Mora, Phys. Rev. Lett. 90, 185002 (2003).

${ }^{13}$ A. R. Karimov, M. Y. Yu, and L. Stenflo, Phys. Plasmas 16, 062313 (2009); 19, 092118 (2012).

${ }^{14}$ F. Robicheaux and J. D. Hanson, Phys. Rev. Lett. 88, 055002 (2002).

${ }^{15}$ I. Blumenfeld, C. E. Clayton, F.-J. Decker, M. J. Hogan, C. Huang, R. Ischebeck, R. Iverson, C. Joshi, T. Katsouleas, N. Kirby, W. Lu, K. A. Marsh, W. B. Mori, P. Muggli, E. Oz, R. H. Siemann, D. Walz, and M. Zhou, Nature 445, 741 (2007).

${ }^{16}$ R. C. Davidson and H. Qin, Physics of Intense Charged Particle Beams in High Energy Accelerators (Imperial College Press, London, 2001).

${ }^{17}$ L. D. Landau and E. M. Lifshitz, Electrodynamics of Continuous Media (Pergamon, Oxford, 1963), p. 103.

${ }^{18}$ H. Luo and M. Y. Yu, Phys. Scr. 74, 670 (2006).

${ }^{19}$ P. K. Shukla and A. A. Mamun, Introduction to Dusty Plasma Physics (IOP, London, 2002).

${ }^{20}$ V. N. Tsytovich, N. G. Gousein-zadeb, and G. E. Morfill, Phys. Plasmas 13, 033503 (2006).

${ }^{21}$ A. V. Gurevich, L. V. Pariskaya, and L. P. Pitaievskii, Sov. Phys. JETP 22, 449 (1966).

${ }^{22}$ O. M. Gradov and L. Stenflo, Phys. Rep. 94, 111 (1983).

${ }^{23}$ N. A. Azarenkov and K. N. Ostrikov, Phys. Rep. 308, 333 (1999).

${ }^{24}$ Sh. Amiranashvili, M. Y. Yu, L. Stenflo, G. Brodin, and M. Servin, Phys. Rev. E 66, 046403 (2002).

${ }^{25}$ A. R. Karimov, Physica D 102, 328 (1997).

${ }^{26}$ A. R. Karimov and H. Schamel, Phys. Plasmas 8, 1180 (2001).

${ }^{27}$ M. M. Leroy, Phys. Fluids 26, 2742 (1983).

${ }^{28}$ A. G. Ponomarenko, Physics of Cosmic and Laboratory Plasmas (Nauka, Novosibirsk, 1989).

${ }^{29}$ T. Ditmire, A. Rubenchik, V. V. Mirnov, and D. Ucer, Astrophys. J. Suppl. Ser. 127, 293 (2000).

${ }^{30}$ Ya. B. Zel'dovich and Yu. P. Raizer, Physics of Shock Waves and HighTemperature Hydrodynamic Phenomena (Dover, Mineola, New York, 2002).

${ }^{31}$ D. A. Osipyan, H. B. Nersisyan, and H. H. Matevosyan, Astrophysics 46, 434 (2003).

${ }^{32}$ H. B. Nersisyan, K. A. Sargsyan, D. A. Osipyan, M. V. Sargsyan, and H. H. Matevosyan, Phys. Scr. 84, 065003 (2011).

${ }^{33}$ D. Winske and N. Omidi, Phys. Plasmas 12, 072514 (2005).

${ }^{34}$ R. S. Fletcher, X. L. Zhang, and S. L. Rolston, Phys. Rev. Lett. 96, 105003 (2006).

${ }^{35} \mathrm{H}$. Schlichting, Boundary-Layer Theory (McGraw Hill, New York, 1979).

${ }^{36} \mathrm{E}$. F. Beckenbach and R. Bellman, Inequalities (Springer-Verlag, Berlin, 1961).

${ }^{37}$ L. Stenflo and M. Y. Yu, Phys. Plasmas 9, 5129 (2002).

${ }^{38}$ A. R. Karimov, J. Plasma Phys. 75, 817 (2009).

${ }^{39}$ A. R. Karimov, Phys. Plasmas 20, 052305 (2013).

${ }^{40}$ K. P. Stanyukovich, Nonstationary Motion of Continuous Medium (Gostekhizdat, Moscow, 1955) (in Russian).

${ }^{41}$ D. H. E. Dubin, Phys. Fluids B 5, 295 (1993).

${ }^{42}$ H. R. Lewis, Phys. Fluids 28, 425 (1985).

${ }^{43}$ A. S. Pleshanov, The Theory of Hydrodynamic Stability-Tornadoes (Informenergo, Moscow, 1993) (in Russian).

${ }^{44}$ H. Schamel, Phys. Rep. 392, 279 (2004). 